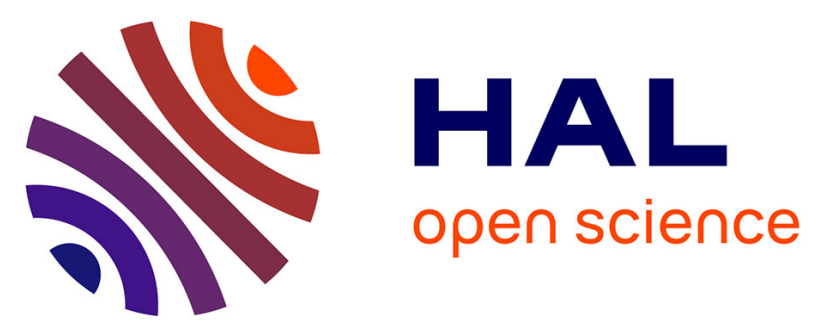

\title{
Synthesis and Crystal Structures of Dinuclear Zinc Complexes with the 3,5-Bis(pyridine-2-yl)pyrazolato Ligand
}

Matthias Westerhausen, Tobias Kloubert, Helmar Görls

\section{- To cite this version:}

Matthias Westerhausen, Tobias Kloubert, Helmar Görls. Synthesis and Crystal Structures of Dinuclear Zinc Complexes with the 3,5-Bis(pyridine-2-yl)pyrazolato Ligand. Journal of Inorganic and General Chemistry / Zeitschrift für anorganische und allgemeine Chemie, 2010, 636 (13-14), pp.2405. 10.1002/zaac.201000168 . hal-00599870

\section{HAL Id: hal-00599870 https://hal.science/hal-00599870}

Submitted on 11 Jun 2011

HAL is a multi-disciplinary open access archive for the deposit and dissemination of scientific research documents, whether they are published or not. The documents may come from teaching and research institutions in France or abroad, or from public or private research centers.
L'archive ouverte pluridisciplinaire HAL, est destinée au dépôt et à la diffusion de documents scientifiques de niveau recherche, publiés ou non, émanant des établissements d'enseignement et de recherche français ou étrangers, des laboratoires publics ou privés. 
Synthesis and Crystal Structures of Dinuclear Zinc Complexes with the 3,5-Bis(pyridine-2-yl)pyrazolato Ligand

\begin{tabular}{|r|l|}
\hline Journal: & Zeitschrift für Anorganische und Allgemeine Chemie \\
\hline Manuscript ID: & zaac.201000168.R1 \\
\hline Watey - Manuscript type: & Article \\
\hline Author: & 19-May-2010 \\
\hline Complete List of Authors: & $\begin{array}{l}\text { Westerhausen, Matthias; Friedrich-Schiller-Universitaet, Institut } \\
\text { fÂd uer Anorganische und Analytische Chemie } \\
\text { Kloubert, Tobias } \\
\text { Görls, Helmar }\end{array}$ \\
\hline Keywords: & zinc, metallation reaction, pyrazolate, crystal structure \\
\hline \multicolumn{2}{|c}{} \\
\hline
\end{tabular}

\section{ऽ ScholaroNE" \\ Manuscript Central}




\title{
Synthesis and Crystal Structures of Dinuclear Zinc Complexes with the 3,5- Bis(pyridine-2-yl)pyrazolato Ligand
}

Tobias Kloubert, Helmar Görls, Matthias Westerhausen*

Institut für Anorganische und Analytische Chemie der Friedrich-Schiller-Universität August-Bebel-Straße 2, D-07743 Jena, Germany

Fax: +49 3641 948102, e-mail: m.we @ uni-jena.de

\begin{abstract}
Zincation of 3,5-bis(pyridine-2-yl)pyrazole with $\mathrm{Zn}\left[\mathrm{E}\left(\mathrm{SiMe}_{3}\right)_{2}\right]_{2}$ in tetrahydrofuran yields dimeric 3,5-bis(pyridine-2-yl)pyrazolato zinc bis(trimethylsilyl)amide $(\mathrm{E}=\mathrm{N}, \mathbf{1})$ and bis(trimethylsilyl)methanide $(\mathrm{E}=\mathrm{CH}, 2)$. These complexes crystallize in the space groups $P 2_{1} / \mathrm{n}$ and $P \overline{1}$, respectively. The zinc atoms are in distorted tetrahedral environments. In the solid state the 3,5-bis(pyridine-2-yl)pyrazolato anions act as bridging tridentate bases with one pyridyl group not participating in the coordination behaviour. In solution exchange processes fast on the NMR time scale lead to a single set of resonances for the pyridyl substituents.
\end{abstract}

\section{Introduction}

Dinuclear zinc complexes play an important role in biomimetic models for enzymes and catalysts [1]. Often pyridyl groups are used to mimic N-bound histidine bases. In the course of our investigations of dinuclear zinc complexes with multi-dentate ligands such as 1,2bis(pyridine-2-yl)-1,2-diamidoethanes [2] or bis(2-pyridylmethyl)amides [3] we included studies with tetradentate 3,5-bis(2-pyridyl)pyrazolato anions. Pyrazolyl and imidazolyl units also play a crucial role in bioinorganic chemistry of zinc in order to mimic active sites of zinc enzymes [4]. 3,5-Substituted pyrazolate ligands represent valuable bridging units between two zinc ions with a strong influence of the side arms (length and nature of functional groups) on the catalytic activity [5]. An easily accessible ligand offering pyrazolyl as well as pyridyl functionalities is the well-known 3,5-bis(pyridine-2-yl)pyrazolate anion. This ligand forms complexes with the late $3 \mathrm{~d}$ transition metals from $\mathrm{Cr}$ to $\mathrm{Cu}$ whereas no examples are known for the earlier transition metals [6]. Only hydrates of 3,5-bis(2-pyridyl)pyrazole zinc halide [7] and nitrate [7, 8] and their homologous cadmium derivatives are reported, however, the molecular structures remained unknown. The rigidity of this 3,5-bis(2-pyridyl)pyrazolyl 
ligand enables the formation of dinuclear metal complexes and in some cases even complexes of higher nuclearity via self-assembly [9]. In general, the 3,5-bis(pyridine-2-yl)pyrazolato ligand acts as a bridging ligand and in bidentate fashion to both the metal cations as displayed in Scheme 1. In these complexes the metals often show hexa-coordination in distorted octahedral environments.
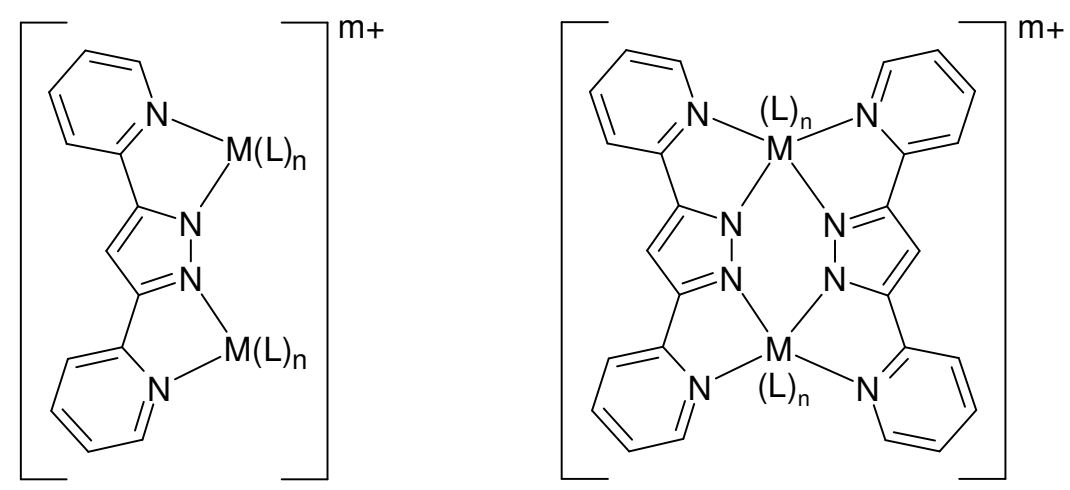

Scheme 1: Common coordination modes of the 3,5-bis(pyridine-2-yl)pyrazolate anion as a bridging ligand. The value of $\mathrm{m}$ depends on the oxidation state on $\mathrm{M}$ and on the charge of $\mathrm{L}$ (which can be solvent molecules and counter anions).

\section{Results and Discussion}

3,5-Bis(pyridine-2-yl)pyrazole was deprotonated with $\mathrm{Zn}\left[\mathrm{E}\left(\mathrm{SiMe}_{3}\right)_{2}\right]_{2}(\mathrm{E}=\mathrm{N}, \mathrm{CH})$ in tetrahydrofuran yielding 3,5-bis(pyridine-2-yl)pyrazolato zinc bis(trimethylsilyl)amide $(\mathrm{E}=$ $\mathrm{N}, \mathbf{1})$ and bis(trimethylsilyl)methanide $(\mathrm{E}=\mathrm{CH}, 2)$, respectively, according to equation 1 . These isotypic compounds were recrystallized from concentrated thf solutions to grow single crystals suitable for X-ray structure determinations.

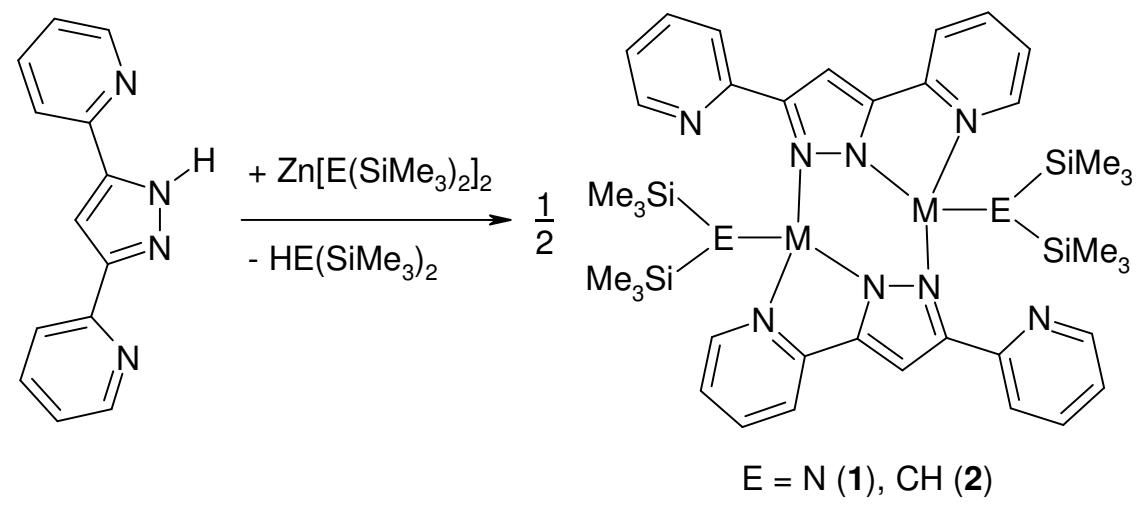

NMR spectroscopic data are summarized in Table 1. The numbering scheme is in accordance with the IUPAC recommendation. The amide or methanide groups show nearly no influence 
Insert Figures 1 and 2

Insert Table 2

Selected structural data of $\mathbf{1}$ and $\mathbf{2}$ are compared in Table 2. In both complexes the endocyclic $\mathrm{Zn1-N1/N2} \mathrm{and} \mathrm{Zn2-N5/N6} \mathrm{bond} \mathrm{lengths} \mathrm{are} \mathrm{significantly} \mathrm{larger} \mathrm{than} \mathrm{the} \mathrm{exocyclic} \mathrm{Zn1-N7}$ and Zn2-N3 distances. Ring formation also leads to narrow N1-Zn1-N2 and N5-Zn2-N6 bond angles of $76.9^{\circ}$. In 1 the $\mathrm{Zn}-\mathrm{N}$ bonds to the bis(trimethylsilyl)amide anions show the smallest values due to a rather large electrostatic attraction. Nevertheless, in $\mathrm{Zn}\left[\mathrm{N}\left(\mathrm{SiMe}_{3}\right)_{2}\right]_{2}$ with a two-coordinate zinc atom significantly smaller $\mathrm{Zn}-\mathrm{N}$ bond lengths are observed $(\mathrm{Zn}-\mathrm{N}$, solid state: 183.3(11) pm [10], gas phase: 182.4(14) pm [11]). The influence of the coordination numbers of $\mathrm{Zn}$ and $\mathrm{N}$ on the $\mathrm{Zn}-\mathrm{N}$ distances is nicely shown for a number of homoleptic zinc amides of the type $\mathrm{Zn}\left[\mathrm{N}\left(\mathrm{SiR}_{3}\right) \mathrm{R}^{\prime}\right]_{2}[12]$ and $\mathrm{Zn}\left(\mathrm{NPh}_{2}\right)_{2}$ [13]. The bulkiness of the $\mathrm{E}\left(\mathrm{SiMe}_{3}\right)_{2}$ ligands $(\mathrm{E}=\mathrm{CH}, \mathrm{N})$ induces steric pressure and enhanced $\mathrm{N}-\mathrm{Zn}-\mathrm{E}$ angles in $\mathbf{1}$ and $\mathbf{2}$. The negative charge of the pyrazolato anions are delocalized within this five-membered aromatic ring which reduces the negative charge on the nitrogen atoms N2, N3 as well as N6, N7. Even though the 3,5-bis(pyridine-2-yl)pyrazolato ligand displays a nearly planar arrangement there is nearly no charge delocalization into the pyridyl moieties; the $\mathrm{C}-\mathrm{C}$ bonds between the aromatic rings show values of approximately $146.6 \mathrm{pm}$ which is characteristic for single 
bonds between $\mathrm{sp}^{2}$ hybridized carbon atoms. The $\mathrm{Zn}-\mathrm{C}$ distances of $201.3 \mathrm{pm}$ are rather large and represent a characteristic value for dialkylzinc with tetra-coordinate metal atoms [14].

\section{Conclusion and Perspective}

In the solid state only one of the pyridyl groups of the anion is bound to zinc. However, in solution an exchange reaction, which is fast on the NMR time scale, leads to two chemically equivalent pyridyl substituents and one set of resonances. The tendency of zinc to favour tetra-coordination leads to unique molecular structures for $\mathbf{1}$ and $\mathbf{2}$ which were unknown for the 3,5-bis(pyridine-2-yl)pyrazolato anions as of yet but they are comparable to complexes of the type $\left[\mathrm{RZn}-\mathrm{N}\left(\mathrm{CH}_{2} \mathrm{Py}\right)_{2}\right]_{2}$ [3]. In these compounds only one set of NMR signals was found for the pyridyl groups but in the solid state only one pyridyl substituent was acting as a Lewis base to the zinc cations.

The catalytic activity of $\left[\mathrm{RZn}-\mathrm{N}\left(\mathrm{CH}_{2} \mathrm{Py}\right)_{2}\right]_{2}$ is based on the fact that the pyridyl groups can act as hemilabile Lewis bases [15]. However, the hydrogen atoms at the $\alpha$-methylene units are rather acidic and deprotonation reactions can occur leading to by-products [3]. This kind of side-reaction is impossible for the 3,5-bis(pyridine-2-yl)pyrazolato ligand. Therefore further investigations are in progress in order to evaluate the catalytic activity of $\mathbf{1}$ and $\mathbf{2}$ because these complexes fulfil the pre-condition that hemilabile ligands offer access to the metal centers.

\section{Experimental}

All manipulations were carried out in an anaerobic argon atmosphere and the solvents were thoroughly dried. IR spectra were recorded with Nujol solutions between $\mathrm{KBr}$ windows. The values of the elemental analysis deviate from theoretical values because weighing of these moisture sensitive compounds was challenging due to the easy loss of cocrystallized thf and also due carbonate formation during combustion even though $\mathrm{V}_{2} \mathrm{O}_{5}$ was added. Starting 3,5bis(pyridine-2-yl)pyrazole [8], $\mathrm{Zn}\left[\mathrm{N}\left(\mathrm{SiMe}_{3}\right)_{2}\right]_{2}$ [16], and $\mathrm{Zn}\left[\mathrm{CH}\left(\mathrm{SiMe}_{3}\right)_{2}\right]_{2}$ [17] were prepared according to literature procedures.

Synthesis of 3,5-bis(pyridine-2-yl)pyrazolato zinc bis(trimethylsilyl)amide (1): 3,5Bis(pyridine-2-yl)pyrazole (570 mg, $5.56 \mathrm{mmol}$ ) were dissolved in $15 \mathrm{ml}$ of thf and cooled to $-30^{\circ} \mathrm{C}$. Zinc bis[bis(trimethylsilyl)amide] $(2.5 \mathrm{~g}, 6.49 \mathrm{mmol})$, dissolved in $15 \mathrm{ml}$ of thf and also cooled to $-30^{\circ} \mathrm{C}$, was added dropwise. The reaction mixture was stirred for further $5 \mathrm{~h}$ 
and then warmed to r.t. The formed precipitate was redissolved at $50^{\circ} \mathrm{C}$ and the solution slowly cooled again to r.t. Crystallization of $\mathbf{1}$ was completed by storing of the reacton mixture at $4{ }^{\circ} \mathrm{C}$ for $2 \mathrm{~d}$. Yield: $980 \mathrm{mg}$ of $\mathbf{1}(2,19 \mathrm{mmol} 85,5 \%)$. Dec. above $238^{\circ} \mathrm{C} .-1 \mathrm{H}$ NMR $\left(\left[D_{8}\right]\right.$ THF $) \delta=8.93\left(d,{ }^{3} \mathcal{J}\left(\mathrm{H}^{8,14}, \mathrm{H}^{9,15}\right)=4.8 \mathrm{~Hz}, 2 \mathrm{H}, \mathrm{Pyr}^{8,14}\right) ; 7.83(\mathrm{ddd}$, $\left.{ }^{3} \mathcal{J}\left(\mathrm{H}^{10,16}, \mathrm{H}^{9,11 ; 15,17}\right)=7.7 \mathrm{~Hz},{ }^{4} \mathcal{J}\left(\mathrm{H}^{10,16}, \mathrm{H}^{8,14}\right)=1.5 \mathrm{~Hz}, 2 \mathrm{H}, \mathrm{Pyr}^{10,16}\right) ; 7.66(\mathrm{~d}$, $\left.{ }^{3} \mathcal{J}\left(\mathrm{H}^{11,17}, \mathrm{H}^{10,16}\right)=7.9 \mathrm{~Hz}, 2 \mathrm{H}, \mathrm{Pyr}^{11,17}\right) ; 7.35\left(\mathrm{ddd},{ }^{3} \mathcal{J}\left(\mathrm{H}^{9,15}, \mathrm{H}^{10,16 ; 8,14}\right)=5.4 \mathrm{~Hz}\right.$ $\left.{ }^{4} \mathcal{J}\left(\mathrm{H}^{9,15}, \mathrm{H}^{11,17}\right)=0.8 \mathrm{~Hz}, 2 \mathrm{H}, \mathrm{Pyr}{ }^{9,15}\right) ; 7.05\left(\mathrm{~s}, 1 \mathrm{H}, \mathrm{H}^{4}\right) ; 0.06\left(\mathrm{~s}, 18 \mathrm{H}, \mathrm{H}^{\mathrm{Me}}\right) .-{ }^{13} \mathrm{C}$ NMR $\left(\left[D_{8}\right]\right.$ THF $) \delta=146.7\left(\mathrm{Pyr}^{8,14}\right) ; 144.7\left(\mathrm{Pyr}^{6,12}\right) ; 136.4\left(\mathrm{Pyr}^{10,16}\right) ; 133.6\left(\mathrm{C}^{3,5}\right) ; 120.1$ (Pyr $\left.{ }^{9,15}\right) ; 117.5$ (Pyr $\left.{ }^{11,17}\right) ; 96.4\left(\mathrm{C}^{4}\right) ; 3.5\left(\mathrm{CMe}^{\mathrm{Me}}\right)$. - IR (Nujol, $\left.\mathrm{cm}^{-1}\right): v=3378$ w, 3115 w, 3053 m, 2920 vs, 2720 w, 2683 m, 2609 w, 2532 w, 2308 m, 1979 m, 1850 m, 1767 m, 1705 m, 1652 w, 1606 vs, 1568 s, 1536 s, 1456 s, 1436 s, 1378s, 1366 w, 1345 s, 1274 w, 1249 vs, 1180 m, 1151s, 1141 m, 1095 m, 1074 m, 1048 w, 1020 w, 1009 w, 987 s, 884 s, 766 s, 738 m, 696 m, 663 s, 639 m, 625 m, 613 m, 552 m, 504 w, 497 m, 470 m, 462w. - MS (El): $m / z(\%)=384^{*}(1.4), 369^{*}(9), 275$ (48), 161 (7), 147 (29), $146(100)\left[\left(\mathrm{Me}_{3} \mathrm{Si}\right)_{2}, \mathrm{HNSi}_{2} \mathrm{Me}_{5}, \mathrm{Si}_{2} \mathrm{Me}_{6}\right]^{+}, 130$ (41), (signals with an asterisk * show the isotopic pattern of $\mathrm{Zn})$. - Elemental analysis $\left(\mathrm{C}_{19} \mathrm{H}_{27} \mathrm{~N}_{5} \mathrm{Si}_{2} \mathrm{Zn}(\right.$ thf $\left.), 519.13\right)$ : calcd.: C 53.11, H 6.98, N 13.46; found: C 52.28, H 6.16, N 13.62.

Synthesis of 3,5-bis(pyridine-2-yl)pyrazolato zinc bis(trimethylsilyl)methanide (2): A solution of zinc bis[bis(trimethylsilyl)methanide] (400 mg, $1.04 \mathrm{mmol})$ in $5 \mathrm{ml}$ of toluene was dropped into a solution of 3,5-bis(pyridine-2-yl)pyrazole $(228 \mathrm{mg}, 1.03 \mathrm{mmol})$ in $5 \mathrm{ml}$ of thf. Then the reaction mixture was heated to $60{ }^{\circ} \mathrm{C}$ for $30 \mathrm{~min}$ and thereafter slowly cooled to r.t. The product crystallized in the shape of colorless cubes. Yield: $420 \mathrm{mg}$ of $\mathbf{2}(0,94 \mathrm{mmol}, 91 \%)$. Dec. above $260^{\circ} \mathrm{C} .-{ }^{1} \mathrm{H}$ NMR $\left(\left[\mathrm{D}_{8}\right] \mathrm{THF}\right) \delta=8.92\left(\mathrm{~d},{ }^{3} \mathcal{J}\left(\mathrm{H}^{8,14}, \mathrm{H}^{9,15}\right)=5.0 \mathrm{~Hz}, 2 \mathrm{H}\right.$, Pyr $\left.{ }^{8,14}\right) ; 7.78\left(\mathrm{ddd},{ }^{3} \mathcal{J}\left(\mathrm{H}^{10,16}, \mathrm{H}^{9,11 ; 15,17}\right)=7.1 \mathrm{~Hz},{ }^{4} \mathcal{J}\left(\mathrm{H}^{10,16}, \mathrm{H}^{8,14}\right)=1.8 \mathrm{~Hz}, 2 \mathrm{H}, \mathrm{Pyr}^{10,16}\right)$; 
$7.65\left(\mathrm{~d},{ }^{3} \mathcal{J}\left(\mathrm{H}^{11,17}, \mathrm{H}^{10,16}\right)=8.0 \mathrm{~Hz}, 2 \mathrm{H}, \mathrm{Pyr}^{11,17}\right) ; 7.29\left(\mathrm{ddd},{ }^{3} \mathcal{J}^{9} \mathrm{H}^{9,15}, \mathrm{H}^{10,16 ; 8,14}\right)=6.6 \mathrm{~Hz}$ $\left.{ }^{4} J\left(\mathrm{H}^{9,15}, \mathrm{H}^{11,17}\right)=1.6 \mathrm{~Hz}, 2 \mathrm{H}, \mathrm{Pyr}^{9,15}\right) ; 7.02\left(\mathrm{~s}, 1 \mathrm{H}, \mathrm{H}^{4}\right) ; 0,10\left(\mathrm{~s}, 1 \mathrm{H}, \mathrm{H}^{19}\right) ;-0.14(\mathrm{~s}, 18 \mathrm{H}$, HMe). $-{ }^{13} \mathrm{C}-N M R\left(\left[D_{8}\right]\right.$ THF $) \delta=152.4\left(\mathrm{Pyr}^{6,12}\right) ; 151.1\left(\mathrm{Pyr}^{8,14}\right) ; 138.9\left(\mathrm{Pyr}^{10,16}\right) ; 122.7$ (Pyr $\left.{ }^{9,15}\right) ; 120.6$ (Pyr $\left.{ }^{11,17}\right) ; 109.6\left(C^{3,5}\right) ; 100.0\left(C^{4}\right) ; 4.9\left(C^{M e}\right) ; 1.3\left(C^{19}\right)$. - IR (Nujol, cm1): $v=3556 \mathrm{vw}, 3118$ w, 3046 w, 2852 s, 2722 w, 2667 w, 2309 vw, 1976 w, 1912 w, 1842 w, 1766 w, 1686 w, 1649 w, 1606 vs, 1590 w, 1565 s, 1533 s, 1454 vs, 1435 vs, 1377 s, 1343 m, 1276 m, 1255 w, 1236 vs, 1150 m, 1135 m, 1095 w, 1072 m, 1049 m, 1027 s, 1019 s, 1007 s, 989 m, 974 m, 897 s, 853 s, 801 m, 765 s, 742 s, 708 w, 695 w, 671 s, 638 m, 622 m, 609 m, 550 m, 497 m, 476 w, 467 m. -MS (EI): $m / z(\%)=444^{*}(28)[\mathrm{M}]^{+}, 429^{*}(100)\left[\mathrm{M}-\mathrm{CH}_{3}\right]^{+}, 367^{*}(8)[\mathrm{M}-\mathrm{Pyridyl}]^{\mathrm{H}+}, 357^{*}(11)[429 \mathrm{H}-$ $\left.\mathrm{SiMe}_{3}\right]^{\mathrm{H}+}, 335(23), 286^{*}(12), 273(16), 145(34), 129(42)$, (signals with an asterisk * show the isotopic pattern of $\mathrm{Zn})$. - Elemental analysis $\left(\mathrm{C}_{20} \mathrm{H}_{28} \mathrm{~N}_{4} \mathrm{Si}_{2} \mathrm{Zn}, 446.05\right)$ : calcd.: C 53.74, H 6.54, N 12.53; found: C 53.56, H 6.35, N 12.61.

Crystal structure determination: The intensity data for the compounds were collected on a Nonius KappaCCD diffractometer, using graphite-monochromated $M o K_{\alpha}$ radiation [18, 19]. The structures were solved by Direct Methods (SHELXs [20]) and refined by full-matrix leastsquares techniques against $F_{\mathrm{o}}^{2}$ (SHELXL-97 [21]). All hydrogen atoms were included at calculated positions with fixed thermal parameters. All non-disordered, non-hydrogen atoms were refined anisotropically [21]. Crystallographic data as well as structure solution and refinement details are summarized in Table 3. XP (Siemens Analytical X-ray Instruments, Inc.) was used for structure representations.

Insert Table 3 


\section{Acknowledgement}

This work was generously supported by the Deutsche Forschungsgemeinschaft (DFG, BonnBad Godesberg, Germany). We are also grateful to the Fonds der Chemischen Industrie (Frankfurt/Main, Germany) for supporting our research.

Supporting Information available: Crystallographic data (excluding structure factors) has been deposited with the Cambridge Crystallographic Data Centre as supplementary publication CCDC-773037 for 1, and CCDC-773037 for 2. Copies of the data can be obtained free of charge on application to CCDC, 12 Union Road, Cambridge CB2 1EZ, UK [E- mail: deposit@ccdc.cam.ac.uk].

[1] J. Weston, Chem. Rev. 2005, 105, 2151-2174.

[2] a) M. Westerhausen, T. Bollwein, N. Makropoulos, T. M. Rotter, T. Habereder, M. Suter, H. Nöth, Eur. J. Inorg. Chem. 2001, 851-857; b) M. Westerhausen, T. Bollwein, M. Warchhold, H. Nöth, Z. Anorg. Allg. Chem. 2001, 627, 1141-1145; c) M. Westerhausen, T. Bollwein, N. Makropoulos, S. Schneiderbauer, M. Suter, H. Nöth, P. Mayer, H. Piotrowski, K. Polborn, A. Pfitzner, Eur. J. Inorg. Chem. 2002, 389-404; d) M. Westerhausen,, T. Bollwein, K. Karaghiosoff, S. Schneiderbauer, M. Vogt, H. Nöth, Organometallics 2002, 21, 906-911.

[3] a) M. Westerhausen, A. N. Kneifel, I. Lindner, J. Grčić, H. Nöth, Z. Naturforsch. 2004, 59b, 161-166; b) E. Jaime, A. N. Kneifel, M. Westerhausen, J. Weston, J. Organomet. Chem. 2008, 693, 1027-1037.

[4] a) H. Vahrenkamp, Dalton Trans. 2007, 4751-4759; b) N. Burzlaff in H.-B. Kraatz, N. Metzler-Nolte (eds.): Concepts and Models in Bioinorganic Chemistry, Weinheim: WileyVCH, 2006; chap. 17, pp. 397-431; c) G. Parkin, Chem. Rev. 2004, 104, 699-767.

[5] F. Meyer, Eur. J. Inorg. Chem. 2006, 3789-3800.

[6] J. Klingele, S. Dechert, F. Meyer, Coord. Chem. Rev. 2009, 253, 2698-2741. See also: a) S. Trofimenko, Chem. Rev. 1972, 72, 497-509; b) A. P. Sadimenko, S. S. Basson, Coord. Chem. Rev. 1996, 147, 247-297; c) R. Mukherjee, Coord. Chem. Rev. 2000, 203, 151-218; d) A. L. Gavrilova, B. Bosnich, Chem. Rev. 2004, 104, 349-383 .

[7] J. Casabó, J. Pons, K. S. Siddiqi, F. Teixidor, E. Molins, C. Miravitlles, J. Chem. Soc., Dalton Trans. 1989, 1401-1403. 
[8] J. Pons, X. López, E. Benet, J. Casabó, F. Teixidor, F. J. Sanchez, Polyhedron 1990, 9, 2839-2845.

[9] a) J. I. van der Vlugt, S. Demeshko, S. Dechert, F. Meyer, Inorg. Chem. 2008, 47, 15761585; b) J.-Z. Hou, M. Li, Z. Li, S.-Z. Zhan, X.-C. Huang, D. Li, Angew. Chem. Int. Ed. 2008, 47, 1711-1714; c) J. Pons, F. J. Sanchez, J. Casabó, A. Alvarez-Larena, J. F. Pinella, J. Ros, Inorg. Chem. Commun. 2003, 6, 833-836.

[10] G. Markgraf, H.-W. Lerner, M. Bolte, M. Wagner, Z. Anorg. Allg. Chem. 2004, 630, 217-218.

[11] A. Haaland, K. Hedberg, P. P. Power, Inorg. Chem. 1984, 23, 1972-1975.

[12] H. Schumann, J. Gottfriedsen, S. Dechert, F. Girgsdies, Z. Anorg. Allg. Chem. 2000, 626, 747-758.

[13] M. A. Putzer, A. Dashti-Mommertz, B. Neumüller, K. Dehnicke, Z. Anorg. Allg. Chem. 1998, 624, 263-266.

[14] See e.g.: M. Melnik, J. Skoršepa, K. Györyová, C. E. Holloway, J. Organomet. Chem. 1995, 503, 1-9.

[15] a) M. Kahnes, J. Richthof, H. Görls, D. Escudero, L. González, M. Westerhausen, J. Organomet. Chem. 2010, 695, 280-290; b) M. Kahnes, H. Görls, L. González, M. Westerhausen, Organometallics 2010, 29, submitted.

[16] a) H. Bürger, W. Sawodny, U. Wannagat, J. Organomet. Chem. 1965, 3, 113-120; b) M. Bochmann, G. Bwembya, K. J. Webb, Inorg. Synth. 1997, 31, 19-23.

[17] M. Westerhausen, B. Rademacher, W. Poll, J. Organomet. Chem. 1991, 421, 175-188.

[18] COLLECT, Data Collection Software; Nonius B.V., Netherlands, 1998.

[19] „Processing of X-Ray Diffraction Data Collected in Oscillation Mode“:Z. Otwinowski, W. Minor in C. W. Carter, R. M. Sweet (eds.): Methods in Enzymology, Vol. 276, Macromolecular Crystallography, Part A, pp. 307-326, Academic Press 1997.

[20] G. M. Sheldrick, Acta Crystallogr. 1990, A46, 467-473.

[21] G. M. Sheldrick, SHELXL-97 (Release 97-2), University of Göttingen, Germany, 1997. 
Table 1: NMR data of 3,5-bis(pyridine-2-yl)pyrazolato zinc bis(trimethylsilyl)amide $(\mathrm{E}=\mathrm{N}$, 1) and bis(trimethylsilyl)methanide $(\mathrm{E}=\mathrm{CH}, 2)\left(\left[\mathrm{D}_{8}\right]\right.$ thf, $\left.25^{\circ} \mathrm{C}\right)$.

\begin{tabular}{|c|c|c|c|c|}
\hline & \multicolumn{2}{|c|}{$\mathbf{1}(\mathrm{E}=\mathrm{N})$} & \multicolumn{2}{|c|}{$2(\mathrm{E}=\mathrm{CH})$} \\
\hline & ${ }^{1} \mathbf{H}$ & ${ }^{13} \mathrm{C}\left\{{ }^{1} \mathrm{H}\right\}$ & ${ }^{1} \mathbf{H}$ & ${ }^{13} \mathrm{C}\left\{{ }^{1} \mathrm{H}\right\}$ \\
\hline $\mathrm{C} 3 / 5$ & - & 133.6 & - & 109.6 \\
\hline $\mathrm{C} 4-\mathrm{H}$ & 7.05 & 96.4 & 7.02 & 100.0 \\
\hline C6/12 & - & 144.7 & - & 152.4 \\
\hline C8/14-H & 8.93 & 146.7 & 8.92 & 151.1 \\
\hline C9/15-H & 7.35 & 120.1 & 7.29 & 122.7 \\
\hline C10/16-H & 7.83 & 136.4 & 7.78 & 138.9 \\
\hline C11/17-H & 7.66 & 117.5 & 7.65 & 120.6 \\
\hline $\mathrm{CHSi}_{2}$ & - & - & 0.10 & 1.3 \\
\hline $\mathrm{SiMe}_{3}$ & 0.06 & 3.5 & -0.14 & 4.9 \\
\hline
\end{tabular}


Table 2: Structural parameters of the metal environments in $\mathbf{1}$ and $\mathbf{2}$ (bond lengths [pm] and angles [deg.]).

\begin{tabular}{lll}
\hline & $\mathbf{1}(\mathrm{E}=\mathrm{N})$ & $\mathbf{2}(\mathrm{E}=\mathrm{CH})$ \\
\hline Zn1-N1 & $211.3(2)$ & $213.1(3)$ \\
Zn1-N2 & $213.3(2)$ & $209.3(2)$ \\
Zn1-N7 & $201.0(2)$ & $205.7(3)$ \\
Zn1-E & $192.3(2)$ & $201.2(3)$ \\
Zn2-N3 & $203.1(2)$ & $202.4(3)$ \\
Zn2-N5 & $212.3(2)$ & $214.0(2)$ \\
Zn2-N6 & $208.6(2)$ & $212.1(3)$ \\
Zn2-E & $192.8(2)$ & $201.3(3)$ \\
E-Si1 & $170.6(2)$ & $184.9(3)$ \\
E-Si2 & $171.6(2)$ & $184.7(3)$ \\
E-Si3 & $170.9(2)$ & $185.2(3)$ \\
E-Si4 & $170.9(2)$ & $185.0(3)$ \\
N1-Zn1-N2 & $76.72(7)$ & $77.13(10)$ \\
N1-Zn1-N7 & $118.83(8)$ & $116.89(19)$ \\
N1-Zn1-E & $112.74(8)$ & $120.48(11)$ \\
N2-Zn1-N7 & $87.04(7)$ & $88.77(10)$ \\
N2-Zn1-E & $123.67(8)$ & $120.32(11)$ \\
N7-Zn1-E & $124.86(8)$ & $119.61(12)$ \\
N3-Zn2-N5 & $120.61(7)$ & $119.79(10)$ \\
N3-Zn2-N6 & $87.85(8)$ & $88.73(10)$ \\
N3-Zn2-E & $126.17(8)$ & $119.09(12)$ \\
N5-Zn2-N6 & $76.92(7)$ & $76.76(10)$ \\
N5-Zn2-E & $108.52(8)$ & $118.23(12)$ \\
N6-Zn2-E & $125.47(8)$ & $121.31(12)$ \\
\hline
\end{tabular}


Table 3: Crystal data and refinement details for the X-ray structure determinations of $\mathbf{1}$ and 2.

\begin{tabular}{|c|c|c|}
\hline & $\mathbf{1}(\mathrm{E}=\mathrm{N})$ & $2(\mathrm{E}=\mathrm{CH})$ \\
\hline Formula & $\mathrm{C}_{38} \mathrm{H}_{54} \mathrm{~N}_{10} \mathrm{Si}_{4} \mathrm{Zn}_{2}, 2\left(\mathrm{C}_{4} \mathrm{H}_{8} \mathrm{O}\right)$ & $\mathrm{C}_{40} \mathrm{H}_{56} \mathrm{~N}_{8} \mathrm{Si}_{4} \mathrm{Zn}_{2}, 1.5\left(\mathrm{C}_{4} \mathrm{H}_{8} \mathrm{O}\right)$ \\
\hline $\mathrm{FW}\left(\mathrm{g} \cdot \mathrm{mol}^{-1}\right)$ & 1038.22 & 1000.18 \\
\hline$T / \mathrm{K}$ & $-140(2)$ & $-90(2)$ \\
\hline Crystal system & monoclinic & triclinic \\
\hline Space group & $P 2{ }_{1} / \mathrm{n}$ & $P \overline{1}$ \\
\hline$a / \AA$ & $13.3555(3)$ & $13.7044(4)$ \\
\hline$b / \AA$ & $26.3010(5)$ & $14.1141(5)$ \\
\hline$c / \AA$ & $15.1101(2)$ & $15.5526(6)$ \\
\hline$\alpha /^{\circ}$ & 90 & $74.086(1)$ \\
\hline$\beta /^{\circ}$ & $95.157(1)$ & $70.244(2)$ \\
\hline$\gamma /^{\circ}$ & 90 & $85.002(2)$ \\
\hline$V / \AA^{3}$ & $5286.13(17)$ & $2722.64(16)$ \\
\hline$Z$ & 4 & 2 \\
\hline$\sigma\left(\mathrm{g} \cdot \mathrm{cm}^{-3}\right)$ & 1.305 & 1.220 \\
\hline$\mu\left(\mathrm{cm}^{-1}\right)$ & 10.44 & 10.09 \\
\hline Measured data & 35385 & 19659 \\
\hline Data with $I>2 \sigma(I)$ & 8038 & 8875 \\
\hline unique data / $R_{\text {int }}$ & $12098 / 0.0621$ & $12342 / 0.0331$ \\
\hline $\mathrm{w} R_{2}\left(\text { all data, on } F^{2}\right)^{\mathrm{a})}$ & 0.0929 & 0.1512 \\
\hline$R_{1}(I>2 \sigma(I))^{\mathrm{a})}$ & 0.0407 & 0.0484 \\
\hline$s^{\mathrm{b})}$ & 0.995 & 1.013 \\
\hline Res. dens./e $\cdot \AA^{-3}$ & $0.686 /-0.417$ & $1.039 /-0.547$ \\
\hline CCDC No. & 773037 & 773038 \\
\hline
\end{tabular}

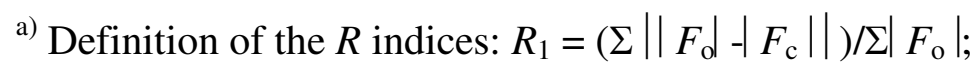
$w R_{2}=\left\{\Sigma\left[w\left(F_{\mathrm{o}}{ }^{2}-F_{\mathrm{c}}\right)^{2}\right] / \Sigma\left[w\left(F_{\mathrm{o}}{ }^{2}\right)^{2}\right]\right\}^{1 / 2}$ with $w^{-1}=\sigma^{2}\left(F_{\mathrm{o}}{ }^{2}\right)+(a P)^{2}+b P ; P=\left[2 F_{\mathrm{c}}{ }^{2}+\operatorname{Max}\left(F_{\mathrm{o}}{ }^{2}\right)\right] / 3 ;$ b) $S=\left\{\Sigma\left[w\left(F_{\mathrm{o}}^{2}-F_{\mathrm{c}}{ }^{2}\right)^{2}\right] /\left(N_{\mathrm{o}}-N_{\mathrm{p}}\right)\right\}^{1 / 2}$. 
Figure 1: Molecular structure and numbering scheme of 3,5-bis(pyridine-2-yl)pyrazolato zinc bis(trimethylsilyl)amide (1). The ellipsoids represent a probability of 40\%, $\mathrm{H}$ atoms are neglected for clarity reasons. 
Figure 2: Molecular structure and numbering scheme of 3,5-bis(pyridine-2-yl)pyrazolato zinc bis(trimethylsilyl)methanide (2). The ellipsoids represent a probability of $40 \%$, hydrogen atoms are omitted. 

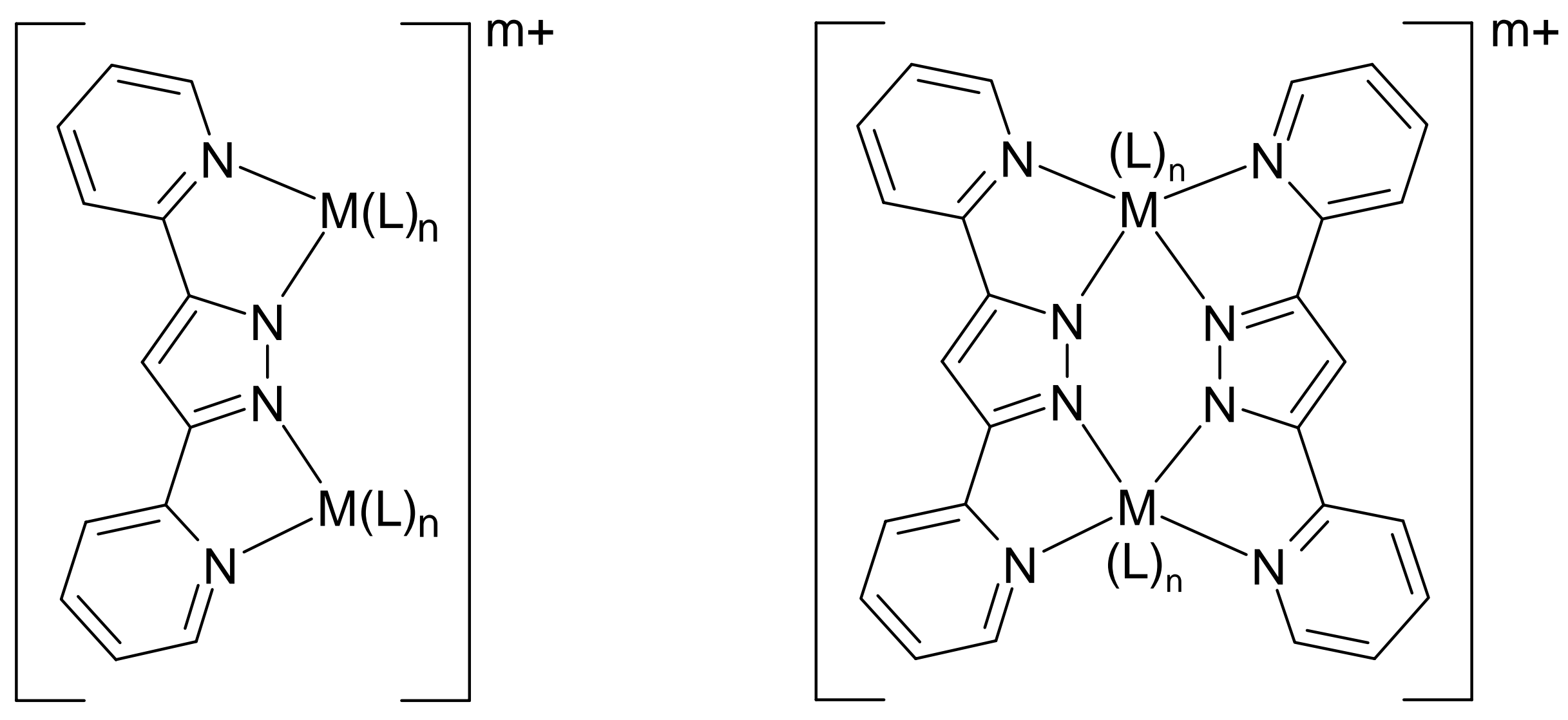


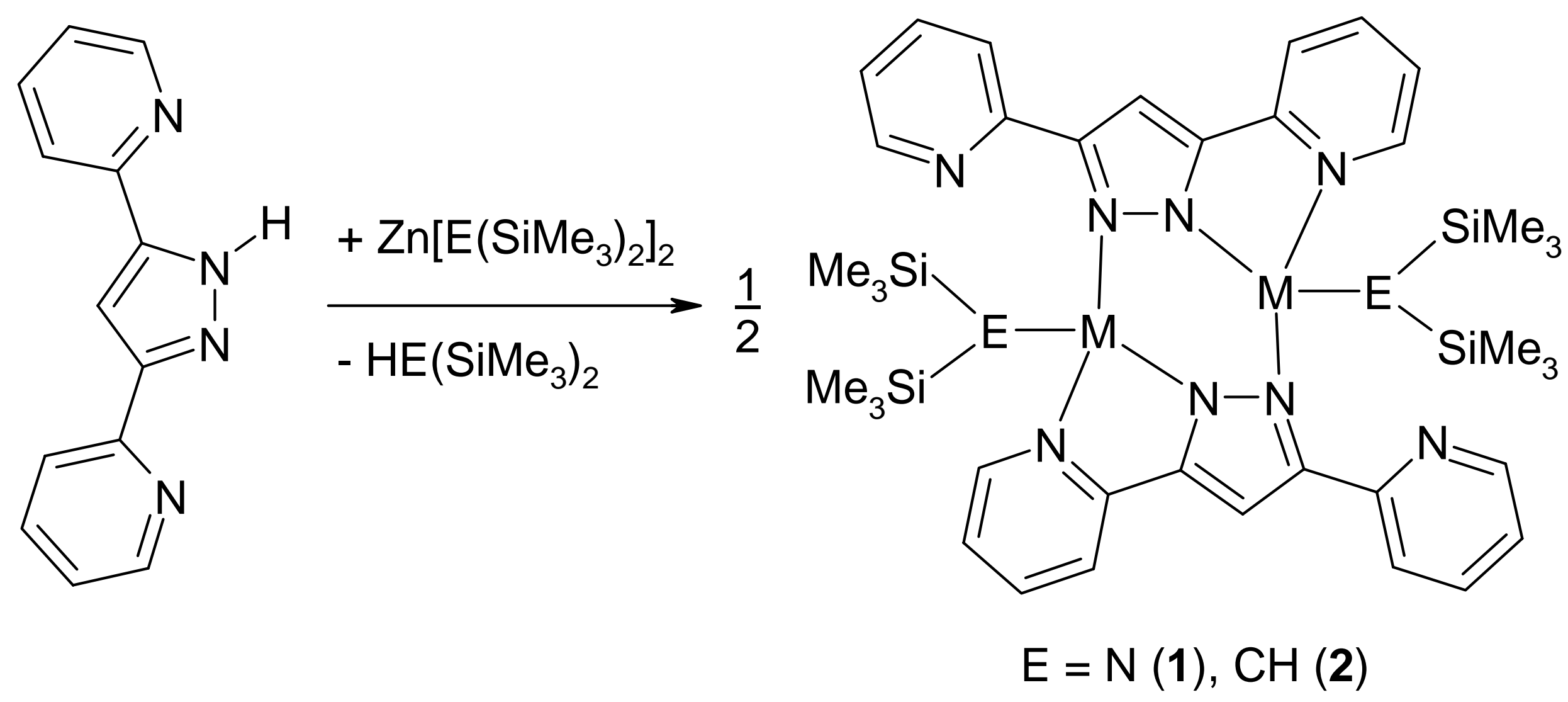




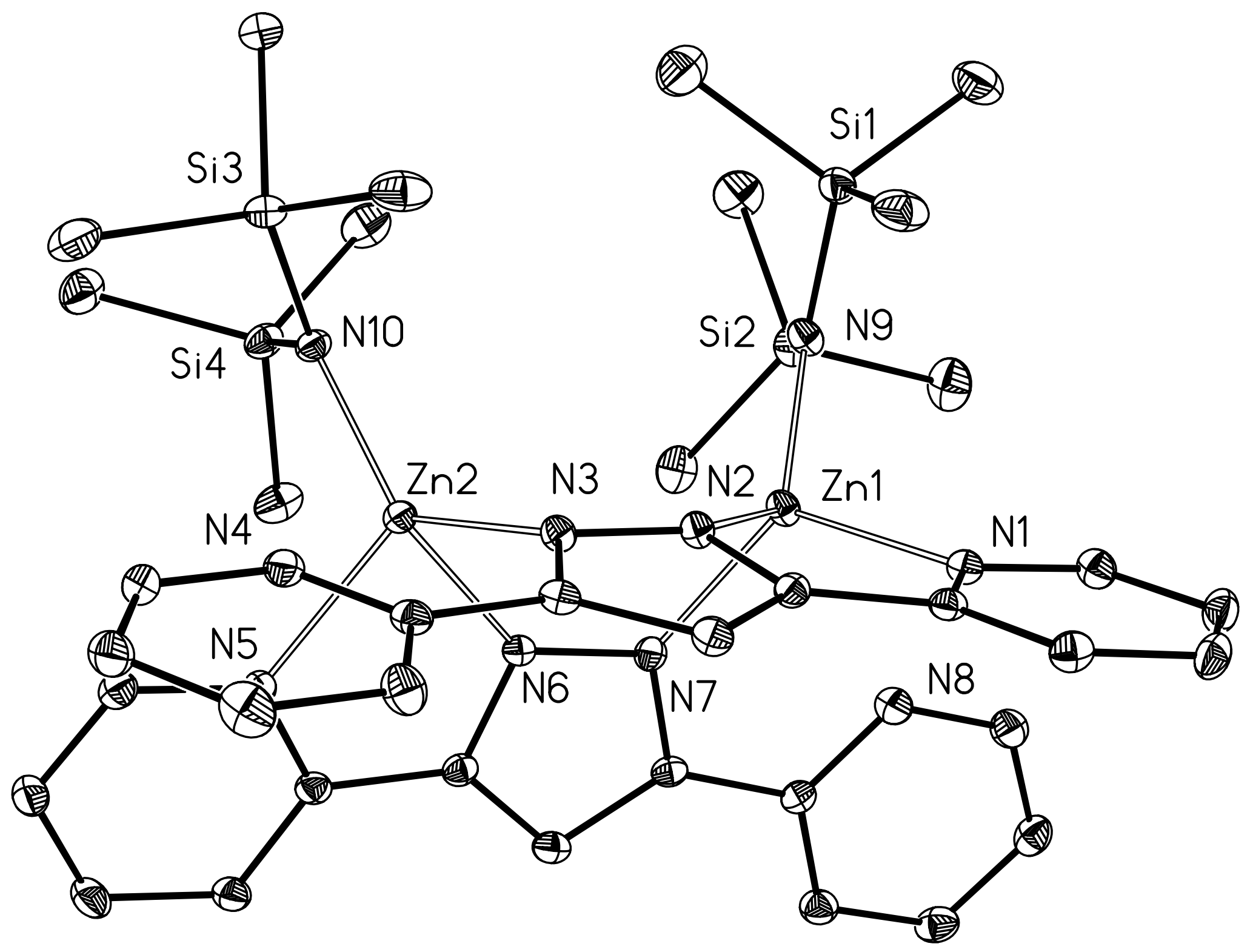




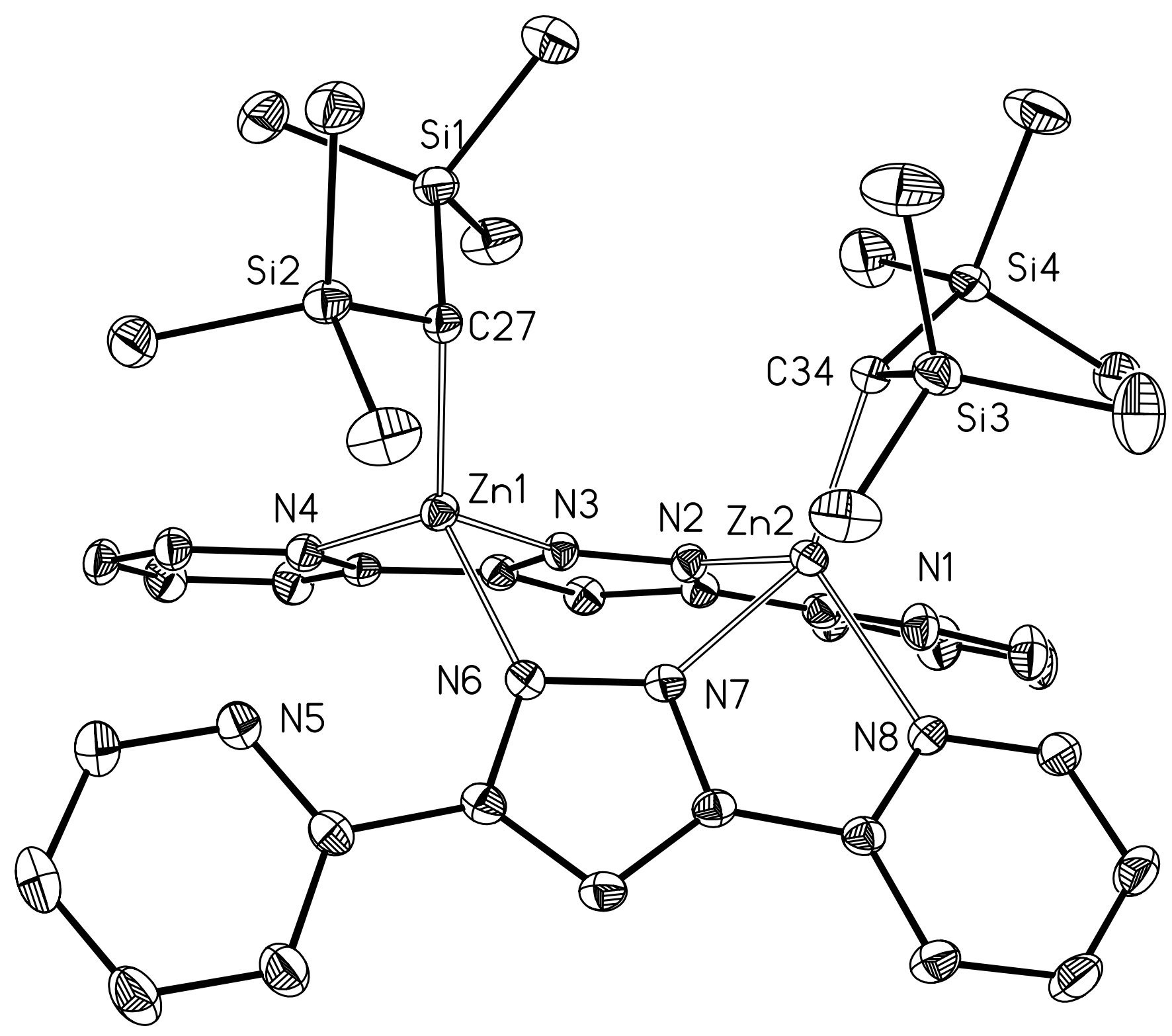

